found this not to be the case in subjects undergoing repeat methionine loading, but have not addressed this explicitly in our study.

In summary, our data show that levels of both total and non-protein-bound homocyst (e)ine are approximately $25 \%$ lower at the time hepatic proteins display an acute phase response. If fasting homocyst ( $e$ )ine is being measured in the assessment of a patient with coronary artery disease, testing is best deferred for $\geq 7$ days after $A M I$.
1. Lindgren A, Brattström L, Norrving B, Bultberg B, Anderson A, Johansson B. Plasma homocysteine in the acute and convalescent phases after stroke. Stroke 1995;26:795-800.

2. Vethoef $P$, Stampfer M, Buring J, Gaziano J, Allen R, Reynolds R, Kok F, Hennekens $\mathrm{C}$, Willett $\mathrm{W}$. Homocysteine metabolism and risk of myocardial infarction: relationship with vitamins $\mathrm{B}_{6}, \mathrm{~B}_{12}$ and folate. Am J Epidemiol 1996; in press.

3. Young B, Gleeson M, Cripps AW. C-reactive protein: a critical review. $\mathrm{Pa}$ thology 1991;23:118-124.

4. Wiley V, Dudman N, Wilcken D. Free and protein-bound homocysteine and cysteine in cystathionime $\beta$-synthase deficiency: interactions during short- and long-term changes in plasma concentrations. Metabolism 1989;38:734-739.

5. Ubbink JB, Vermaak WJH, Bissport S. Rapid high-performance liquid chromatographic assay for total homocysteine levels in human serum. J Chromatogr 1991;565:441-446.

\title{
Usefulness of Three-Dimensional Reconstruction for Interpretation and Quantitative Analysis of Intracoronary Ultrasound During Stent Deployment
}

\author{
Robert Gil, MD, Clemens von Birgelen, MD, Francesco Prati, MD, Carlo Di Mario, MD, PhD, \\ Jurgen Ligthart, and Patrick W. Serruys, MD, PhD
}

ntracoronary ultrasound (ICUS ) - guided stent deployment achieves a significantly larger postprocedural lumen and allows a safe withdrawal of systemic anticoagulation after the procedure with a low incidence of subacute thrombosis, recurrence of clinical symptoms, and restenosis. ${ }^{1}$ However, the review and quantitative analysis from videotape of a conventional ICUS examination (2-dimensional [2-D]) is cumbersome, time-consuming, and requires expert image interpretation, with risk of considerable interobserver disagreement, especially during coronary intervention. ${ }^{2}$ In this study, the ICUS measurements obtained after angiographic optimization of stent deployment with a conventional, manual tracing of the lumen are compared with on-line automatic measurements obtained with a new system of 3-dimensional (3-D) ICUS reconstruction.

Forty-nine stents in 33 patients ( 24 men and 9 women, mean age $60 \pm 9$ years) were examined in this study. Self-expanding (19x Wallstent) and balloon-expandable (19x Palmaz-Schatz, 6x Micro-Stent, 3x Cordis, 1x Multi-Link, 1x Gianturco-Roubin) stents were implanted in 14 left anterior descending artery lesions $(\mathrm{n}=17), 1$ left circumflex artery lesion $(\mathrm{n}=$ $2), 12$ right coronary artery lesions $(n=23)$, and 6 saphenous venous graft lesions $(n=7)$. Coronary stenting was performed either as an elective $(n=26)$ or as a bailout procedure $(n=23)$ in restenotic $(n=$ $17)$ and in de novo $(n=32)$ lesions.

From the Intracoronary Imaging Laboratory and Cardiac Catheteri zation Laboratory, Thoraxcenter, Rotterdam, The Netherlands. Dr. Gil is the recipient of the European Society of Cardiology Training Fellowship, 1994 (Sophia Antipolis-Nice), and Dr. von Birgelen is the recipient of a Fellowship from the German Research Society (Bonn). Dr. Serruys' address is: Cardiac Catheterization Laboratory, Division of Cardiology, Thoraxcenter, BD 432, Erasmus University, P.O. Box 1738, 3015 GD Rotterdam, The Netherlands. Manuscript received September 22, 1995; revised manuscript received and accepted November 15, 1995 .
All patients received acetylsalicylic acid $250 \mathrm{mg}$ and heparin 10,000 IU after insertion of the arterial sheath. If necessary, administration of heparin was repeated in order to maintain an activated clotting time of $>300$ seconds.

Quantitative coronary angiography was performed by means of a previously described computer-assisted automatic quantitative analysis system (CAAS II, Pie Medical Data, Maastricht, or ACA, Philips DCI, Eindhoven, The Netherlands, ) using an edge-detection algorithm. ${ }^{3}$

Before stent placement, predilatation with standard balloon angioplasty was performed. For the balloon-expandable stents, a diameter of the delivery balloon matching the angiographic reference lumen diameter was selected. Then all the stents were redilated with a balloon $\geq 0.25 \mathrm{~mm}$ larger than the angiographic reference lumen diameter. Dilatations within the stent were performed at $>14$ atm with short balloons of low compliance (Chubby or Speedino, Schneider Europe, Zurich, Switzerland). The procedural end point was considered the achievement of a uniform lumen of the treated segment, with an intrastent minimal luminal diameter equal to or greater than the user-defined reference diameter. After intracoronary administration of 1 to $3 \mathrm{mg}$ of isosorbide dinitrate, the ICUS examination was performed using a $2.9 \mathrm{Fr}$ monorail system with a 30 $\mathrm{MHz}$ ultrasound transducer (MicroView, CVIS, Sunnyvale, California).

Ultrasound images were recorded continuously on videotape during withdrawal of the ultrasound probe along the stented segment with the use of a motorized pullback device at a constant speed of 1.0 $\mathrm{mm} / \mathrm{s}$. Based on the recorded images, the proximal and distal reference segments were chosen within 5 $\mathrm{mm}$ from the stent endings. The ICUS cross section with the minimal intrastent luminal area was then identified, and the manually traced contour of this lumen area was visibly maintained while scrolling 


\begin{tabular}{|c|c|c|c|c|c|}
\hline & 2-D ICUS & 3-D ICUS & Mean Difference & p Value & r Value \\
\hline $\operatorname{Min}$. LA $\left(\mathrm{mm}^{2}\right)$ & $8.21 \pm 2.38$ & $7.76 \pm 2.50$ & $0.45 \pm 1.47$ & 0.37 & 0.82 \\
\hline Min. ILD (mm) & $2.98 \pm 0.43$ & $2.67 \pm 0.37$ & $.032 \pm 0.36$ & 0.08 & 0.59 \\
\hline Max. ILD (mm) & $3.32 \pm 0.54$ & $3.10 \pm 0.51$ & $0.22 \pm 0.45$ & 0.04 & 0.63 \\
\hline Symmetry index & $0.90 \pm 0.08$ & $0.87 \pm 0.10$ & $0.04 \pm 0.1$ & 0.054 & 0.46 \\
\hline $\begin{array}{l}\text { LA proximal } \\
\text { reference }\left(\mathrm{mm}^{2}\right)\end{array}$ & $11.8 \pm 4.0$ & $11.5 \pm 3.7$ & $0.05 \pm 1.88$ & 0.67 & 0.89 \\
\hline $\begin{array}{l}\text { LA distal } \\
\quad \text { reference }\left(\mathrm{mm}^{2}\right)\end{array}$ & $10.5 \pm 2.8$ & $10.7 \pm 3.4$ & $-0.09 \pm 1.79$ & 0.84 & 0.86 \\
\hline \multicolumn{6}{|c|}{ 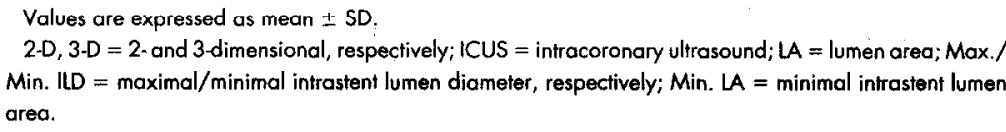 } \\
\hline
\end{tabular}

sectional area assessed by quantitative angiography were $2.99 \pm 0.51$ $\mathrm{mm}$ and $7.23 \pm 2.38 \mathrm{~mm}^{2}$, respectively. Percent diameter stenosis assessed on-line was $-16 \pm 11.4 \%$. Negative percent diameter stenosis was reached in 45 stents ( $92 \%)$.

Of the 49 stents reconstructed, 6 $(12.2 \%)$ needed major manual correction due to a failure of the algorithm used for lumen detection induced by the presence of side branches and shadowing from calcium.

The time required for frame selection and measurement was $4.4 \pm$ 1.5 minutes for the conventional 2 -

through the entire stent length in order to check the correct frame selection. At the same time as this conventional 2-D analysis, the ultrasound images were also acquired with an on-line 3-D reconstruction system that performs an automatic detection of the lumen based on the differences between the pattern of the ultrasound speckle of lumen and coronary wall (EchoQuant Indec Systems Inc., Capitola, California). The accuracy of the automated measurements with this system has been previously validated in an animal model. ${ }^{4}$ The system acquires 255 transverse ultrasonic images in 30 seconds; after a processing time of 105 seconds, the system displays the longitudinal vicw of the analyzed segment $(30 \mathrm{~mm}$ long at $1 \mathrm{~mm} / \mathrm{s}$ pullback speed) and a diagram showing the area and corresponding diameter measurements over the entire reconstructed segment. By scrolling the longitudinal view or the measurement diagram with a cursor, the minimal intrastent lumen crosssectional area can be detected and a reference proximal and distal frame selected. The contours of the minimal intrastent cross-sectional area and of the reference segments can be manually corrected in case of inaccurate automated detection. This 3-D system also displays the reconstructed segment in a cylindric format opened longitudinally (clamshell view).

Ultrasound criteria of the adequacy of stent deployment were derived from the experience of the Milan group ${ }^{1}$ with slight modification. They consisted of: (1) complete apposition of the stent to the vessel wall without protrusion of the struts within the lumen; (2) minimal to maximal intrastent diameter ratio (symmetry index) $>0.7$; and ( 3 ) minimal stent lumen area $\geq 80 \%$ of the average lumen area of the proximal and distal reference segments, or equal to or more than the distal or proximal reference lumen area.

Matched measurements provided by both methods were compared by using a 2-tailed Student's $t$ test for paired data and linear regression analysis. Mean \pm SD difference of the signed difference was calculated as proposed by Bland and Altman. ${ }^{5} \mathrm{~A}$ p value $<0.05$ was considered statistically significant.

After the stenting procedure, the minimal lumen diameter and corresponding minimal luminal cross-
D ICUS examination and $2.6 \pm 0.4$ minutes for the assessment with 3-D ICUS ( $\mathrm{p}<0.05$ ).

Measurements obtained with 2-D and 3-D ICUS are listed in Table I. Intrastent measurements provided by 2-D ICUS were higher than those by 3-D; however, the difference was significant only for the maximal lumen diameter. The best correlation between these 2 methods was found for minimal intrastent cross-sectional area (Figure 1); for Wallstents, however, this correlation was weaker than for other stents $(r=0.63$ vs $r=0.89)$. Both ultrasound methods revealed that the intrastent minimal luminal cross-sectional area was localized at 1 of the endings (within the first or last $2 \mathrm{~mm}$ ) in 31 of 49 analyzed stents $(63 \%)$. There was no significant difference between 2-D and 3-D measurements in cross-sectional lumen area for proximal and distal references $(r=0.89$ and $r=0.86$, respectively). However, the presence of atherosclerotic encroachment of $>50 \%$ in reference segments was found in 20 stents $(40.8 \%)$ by 2-D and in 11 stents $(22.4 \%)$ by 3 -D ICUS.

Two-dimensional ultrasound showed that the quantitative criteria of adequate stent deployment were met in 19 stents $(38.8 \%)$, whereas 3-D ultrasound revealed that these criteria were reached in only 15 of 49 stents (30.6\%) (Figure 2). In 5 of 15 cases $(33.3 \%)$ assessed by $3-\mathrm{D}$ as properly deployed, 2-D ICUS evaluation was different. Both ultrasound methods detected 4 patients $(8.2 \%)$ with protrusion of stent struts within the vessel lumen. In these patients, the symmetry index $<0.7$ was found as additional evidence of stent underexpansion (Figure 3).

Using the information provided by on-line ultrasound measurements, we performed an additional intrastent dilatation in 7 patients $(21.2 \%)$, including all patients with a symmetry index $<0.7$. Nevertheless, in 1 of these patients in whom the GianturcoRoubin stent was implanted as a bailout procedure (because of severe dissection after balloon angioplasty), a subacute stent thrombosis occurred. The patient underwent successful surgery without developing myocardial infarction. 


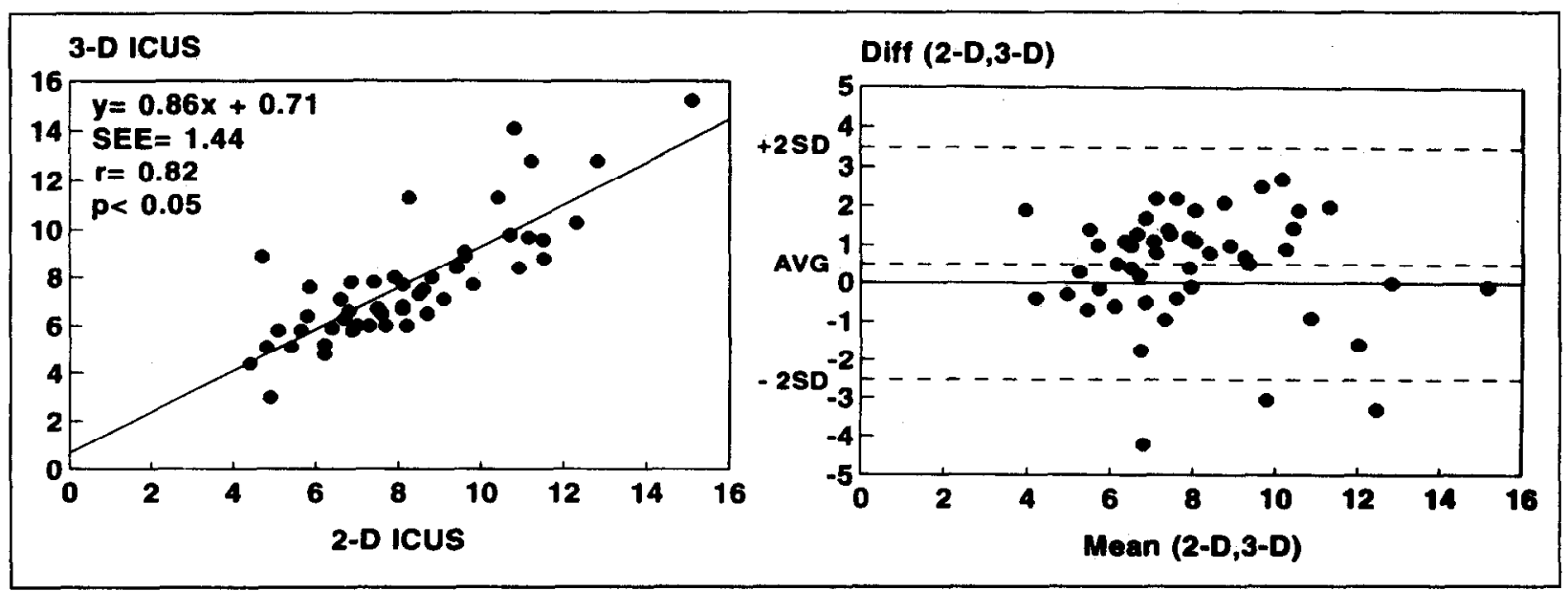

FIGURE 1. Left panel, comparison of minimal intrastent areas obtained with 2- (2-D) and 3- (3-D) dimensional intracoronary ultrasound (ICUS) after angiographic optimization in a studied group of stents. Right panel, the difference between measurements provided by 2 ultrasound modes are plotted against the mean of the 2 appropriate measurements (Diff [2-D,3-D], Mean [2-D,3-D], respectivety). Note that for minimal intrastent cross-sectional luminal area, a very good correlation with an acceptable dispersion of the measurements obtained by both ultrasound methods was found. AVG = average value.

ICUS is a new imaging technique, providing valuable information concerning vessel wall and plaque composition. Based on the results of extensive tests performed in various clinical settings, it appears that ICUS correlates well with histology ${ }^{6}$ and provides accurate and reproducible measurements of lumen and plaque. ${ }^{2}$ Furthermore, it has been proven that this technique is relatively safe ${ }^{7}$ and helpful in the decision-making process. ${ }^{8}$ A report from the group in Milan ${ }^{1}$ clearly showed the inaccuracies of angiographic assessment after stent deployment and the importance of ICUS in the optimization of stent deployment. However, assessment of the adequacy of stent deployment based on analysis of the cross-sectional images acquired during manual withdrawal of the ultrasound catheter is a potential source of error because detection of the narrowest sitc within the stented segment is subjective and may be inaccurate. To circumvent this limitation, we performed ultrasound examinations using a motorized pullback device at constant speed. This was a prerequisite for the on-line application of 3-D ICUS of an analyzed segment. This approach has the potential to assess lumen dimensions and geometry precisely within the stent, and to evaluate the dimensions and vessel wall characteristics of the reference segments. ${ }^{9}$ Furthermore, this method makes the ultrasound measurements more objective, decreases their variability, and may facilitate further interventions.

The fact that $33.3 \%$ of the cases selected by 3 $D$ analysis as properly deployed were missed by 2 D ICUS suggests that during on-line 2-D analysis, the narrowest site within the stent can be missed in a significant number of cases. Comparison of the measurements provided by these 2 methods has shown that systematic overestimation of intrastent dimensions by 2-D ICUS occurs, resulting in a higher percentage of adequately deployed stents than found by 3-D ICUS. The probability of dis- agreement between both ICUS modes in detecting the narrowest intrastent site increases with the length of the stented segment. The lower correlation between 2-D and 3-D ICUS measurements of minimal intrastent luminal area for Wallstents (which are more symmetric but longer than other stents ) supports this thesis. Ultrasound analysis of the reference segments has shown that less diseased segments (but rather with larger lumen areas ) were selected by 3-D ICUS. This fact is reflected by a larger number of patients with significant plaque burden within the reference segments found by 2-D, which further explains the tendency to overestimate the results of stent deployment by 2-D ICUS

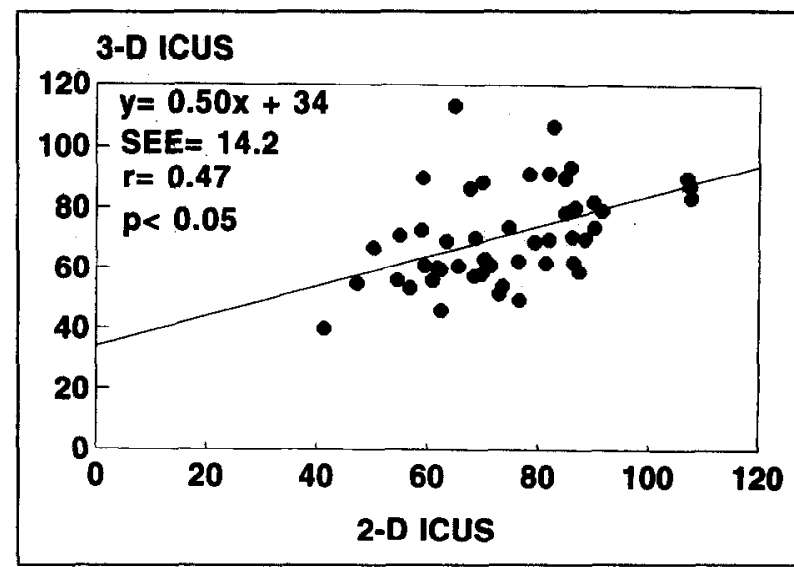

FIGURE 2. Regression analysis of percentage of average reference lumen cross-sectional area measurements (proximal and distall) obtained by 3-dimensional (3-D) (y-axis) and 2-dimensional (2-D) (x-axis) intracoronary ultrasound (ICUS). After angiographic optimization in all cases, average reference lumen area is $\geq \mathbf{4 0} \%$. Nole the discrepancy between measurements oblained with both techniques, with a tendency to overestimation of the postprocedural lumen in most patients by 2 -dimensional ultrasound. 


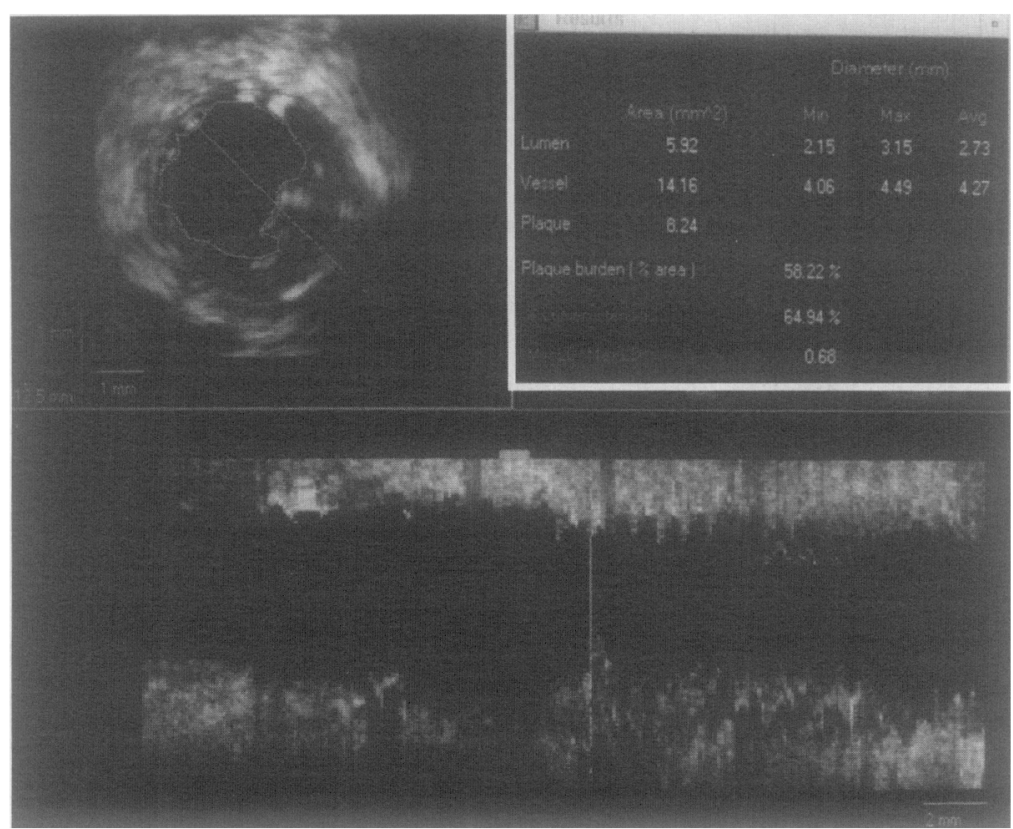

FIGURE 3. Three-dimensional reconstruction of stented segment of left anterior descending artery, clearly showing stent asymmetry and protrusion of its struts within the vessel lumen (at the site of the cursor in the lower panel [vertical linel). Longitudinal display of the reconstructed segment (bottom) shows that the rest of the segment is adequately deployed. The table (right upper comer) provides quantitative measurements of the chosen frame. Avg, Max, and Min = average, maximal, and minimal, respectively; Min LD/Max LD: ratio of minimal to maximal lumen diameters.
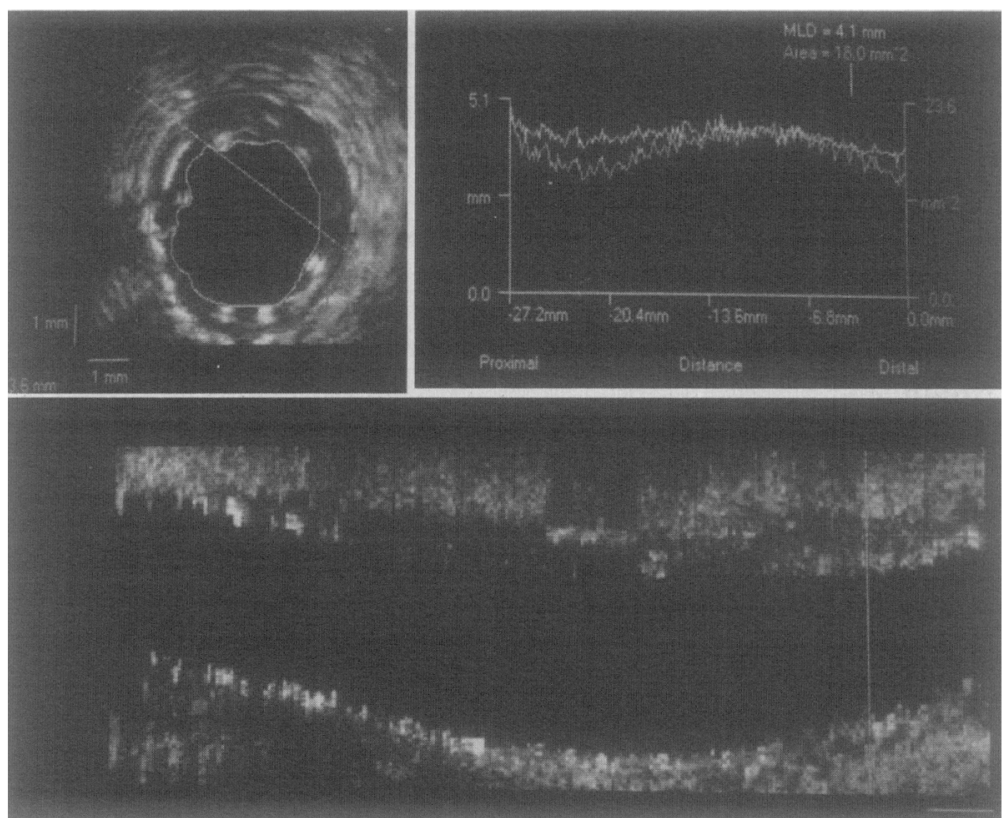

FIGURE 4. Thrse-dimensional roconstruction of right coronary artery showing a fusiform shape of the treated segment with maximal intrastent diameter in its midportion and minimal lumen diameter (MLD) at its ending (vertical cursor in the lower panell. Note the lack of difference in lumen diameter between the most narrowed portion of stent and distal segment of the vessel.
Our data also revealed interesting findings concerning the localization of the minimal intrastent luminal area. The fact that in most patients this parameter was identified at 1 of the stent endings reflects the current approach to stent deployment. According to common practice, a short balloon of low compliance is mainly chosen for additional intrastent dilatation to avoid an injury of the nonstented segments. ${ }^{1}$ As a consequence, a smaller expansion of the stent extremities occurs (Figure 4). This results in a fusiform shape of the stent, which is visible on the longitudinal 3-D display.

In conclusion, on-line 3-D ICUS is feasible during stent implantation, more sensitive than 2-D ICUS in the assessment of optimal stent expansion, and requires a shorter time for analysis.

1. Colombo A, Hall P, Nakamura S, Almagor Y, Maiello L, Martini G, Gaglione A, Goldberg SL, Tobis JS. Intracoronary stenting without anticoagulation accomplished with intravascular guidance. Circulation 1995;91:1676-1688.

2. Hausmann D, Lundkvist A-JS, Friedrich GJ, Mullen WL, Fitzgerald PJ, Yock PG. Intracoronary ultrasound imaging: intraobserver and interobserver variability of morphometric measurements. Am Heart J 1994;128:674-680. 3. Haase J, Di Mario C, Slager CJ, van der Giessen WJ, den Boer A, de Feyter PJ, Reiber JHC, Verdouw PD, Serruys $\mathrm{PW}$. In-vivo validation of on-line and off-line geometric coronary measurements using insertion of stenosis phantoms in porcine coronary arteries. Cathet Cardiovasc Diagn 1992;27:16-27.

4. Hausmain D, Friedrich G, Sudhir K, Mullen WL, Soni B, Fitzgerald PJ, Yock PG. 3-D intravascular ultrasound imaging with automated border detection using $2.9 \mathrm{~F}$ catheters (abstr). I Am Coll Cardiol 1994:23:174A

5. Bland JM, Altman DG. Statistical methods for assessing agreement between two methods of clinical measurement. Lancet 1986;2:307-310.

6. Di Mario C. The SHK, Madretsma S, van Suylen RJ, Wilson RA, Boom N, Serruys PW, Gussenhoven EJ, Roelandt JRTC, Zhong Y, Wenguang $L$. Detection and characterization of vascular lesions by intravascular ultrasound: an in vitro study correlated with histology. $J$ Am Soc Echocardiogr 1992;5:135-146.

7. Hausmann D, Erbel R, Alibelli-Chemarin MJ, Boksch W, Caracciolo E, Cohn JM, Culp SC, Daniel WG, De Scheerder I, DiMario C, et al. The safety of intracoronary ultrasound. A multicenter survey of 2207 examinations. Circulation 1995;91:623-630.

8. Mintz GS, Pichard AD, Kovach JA, Kent KM, Satler LF, Javier SP, Popma JJ, Leon MB. Impact of preintervention intravascular imaging on transcatheter treatment strategies in coronary artery disease. Am $J$ Cardiol 1994;73:423-430.

9. Di Mario C, von Birgelen C, Prati F, Soni B, Li W, Bruining N, de Feyter PJ, Serruys PW, Roelandt JRTC. Three dimensional reconstruction of two-dimensional intracoronary ultrasound: clinical or research tool? Br Heart $J$ 1995; 73 (suppl 2):26-32 